\title{
QT and JT Dispersion in Children With Familial Mediterranean Fever
}

\author{
M. Kürşat FİDANCI, ${ }^{1}$ Ayhan KILIÇ, ${ }^{1}$ Mustafa GÜLGÜN,${ }^{1}$ Gökalp BAŞBOZKURT, ${ }^{2}$ Cengizhan AÇIKEL, ${ }^{3}$ \\ Doğan ŞİMŞEK, ${ }^{4}$ Faysal GÖK, ${ }^{4}$ Erkan DEMİRKAYA, ${ }^{4,5}$ \\ ${ }^{1}$ Department of Pediatric Cardiology, Gülhane Military Medical Faculty, Ankara, Turkey \\ ${ }^{2}$ Department of Pediatrics, Gülhane Military Medical Faculty, Ankara, Turkey \\ ${ }^{3}$ Department of Biostatistics, Gülhane Military Medical Faculty, Ankara, Turkey \\ ${ }^{4}$ Department of Pediatric Rheumatology, Gülhane Military Medical Faculty, Ankara, Turkey \\ ${ }^{5}$ FMF Arthritis Vasculitis and Orphan Disease Research in Pediatric Rheumatology (FAVOR), Ankara, Turkey
}

\begin{abstract}
Objectives: This study aims to determine QT dispersion and JT dispersion, and their relationship with conventional echocardiography values in a group of children with familial Mediterranean fever (FMF).

Patients and methods: The study included 48 FMF patients ( 26 males, 22 females, mean age $11.10 \pm 3.42$ years; range 5 to 18 years) as the FMF patients and 31 healthy children (17 males, 14 females, mean age $9.61 \pm 2.83$ years; range 5 to 17 years) as the healthy controls. Electrocardiography and conventional echocardiography were performed on the FMF patients and healthy controls. Both groups were evaluated with a standard 12-lead electrocardiography. QT, JT and RR distances were measured in both groups. The corrected QT (QTC) and corrected JT (JTc) were calculated. QTcd and corrected JT dispersion (JTCd) were detected.

Results: There was no statistically significant difference between the FMF patients and healthy controls in terms of RR, QT, QTd, QTcd, JT, JTc, JTd, and JTcd measurements and echocardiography parameters. QTc value was higher in the FMF patients than the healthy controls.

Conclusion: QTc value indicates increased ventricular sensitivity and is an important marker of cardiovascular mortality. It has an important effect on sudden cardiac death and arrhythmia. Our study results suggest that electrocardiographic monitoring may be useful in patients with FMF.

Keywords: Cardiac repolarization; Familial Mediterranean fever; JT dispersion; QT dispersion.
\end{abstract}

Familial Mediterranean fever (FMF) is the most prevalent hereditary auto-inflammatory disease (about 100,000 subjects affected worldwide), characterized by recurrent attacks of fever and serositis at short durations (mean 24-72 hours). It is an autosomal recessive disease and is fairly common in Mediterranean countries (Turkey, Armenia, Israel, and North). A few hundred cases have also been reported in Italy and Greece, and more recently in Japan. ${ }^{1-3}$

Inflammation may play an important role in the initiation and progression of atherosclerosis and ischemic cardiac disease. ${ }^{4-6}$ Various clinical and subclinical cardiovascular involvements have been reported in FMF patients., ${ }^{4,5}$ Patients with inflammatory diseases are now considered to have an increased risk of atherosclerotic cardiovascular complications. $^{8}$

Cardiac repolarization abnormalities may be seen due to cardiac involvement in FMF. Variations in the duration of the action potential may create a ventricular repolarization dispersion that may be arrhythmogenic. ${ }^{9}$ This dispersion of repolarization represents the degree of repolarization heterogeneity in the heart, and it has been reported that increased dispersion shows increased susceptibility to complex ventricular tachyarrhythmias and sudden deaths. ${ }^{6,7}$ 
The heterogeneity of ventricular repolarization dispersion is measured by calculating the QT dispersion (QTd). ${ }^{10} \mathrm{JT}$ dispersion (JTd) has been shown to be a better predictor of sudden death in patientswith myocardial infarction. ${ }^{11}$ Consequently, increased heterogeneity of repolarization has been shown in several heart diseases, and has been associated with an increased risk of ventricular tachyarrhythmias. ${ }^{12,13}$

Little is known yet about the influence of children with FMF on the cardiac involvement and heterogeneity of ventricular repolarization, which represents the subclinic inflammation of FMF and is identified on the electrocardiogram by QTd, JTd, and corrected QT (QTcd) and corrected JT dispersions (JTcd). Therefore, in this study, we aimed to determine QTd and JTd, and their relationship with conventional echocardiography values in a group of children with FMF.

\section{PATIENTS AND METHODS}

This study conducted in Department of Pediatric Cardiology in Gulhane Military Medical Academy between March 2012 and March 2013. The study included 48 FMF patients (26 males, 22 females; mean age $11.10 \pm 3.42$ years; range 5 to 18 years), who were in attack-free period and administered regular colchicine therapy, as the FMF patients and 31 healthy children (17 males, 14 females; mean age $9.61 \pm 2.83$ years; range 5 to 17 years) as the healthy controls. All FMF patients were diagnosed according to the clinical criteria outlined by Livneh et al. ${ }^{14}$ The healthy controls consisted of 31 healthy volunteers, comparable to the FMF patients for age and sex. Patients with diabetes mellitus, hypertension, chronic renal failure, chronic liver disease, malignancy, amyloidosis, active infectious disease, cardiomyopathy, rhythm abnormalities, and congenital or acquired heart disease were excluded. All the patients were in sinus rhythm, and none of them were taking medications such as antiarrhythmics, tricyclic antidepressants, antihistamines, or antipsychotics. Clinical and laboratory assessments of FMF patients were performed during an attack-free period. The study was approved by the local ethics committee of the research hospital.

The FMF patients and healthy controls were evaluated with a standard 12-lead electrocardiography (ECG). QT, JT, and RR distances were measured in both groups. The QTc and JTc were calculated. QTcd and JTcd were determined.

The 12-lead ECG was recorded at a paper speed of $25 \mathrm{~mm} / \mathrm{second}$, and a gain of $10 \mathrm{~mm} / \mathrm{mV}$ in the supine position. Electrodes were placed in anatomical positions according to routine procedure. ECG strips were recorded for 10 seconds with a standard device. ECGs of inadequate quality were repeated. QT intervals were measured manually from the point where the $\mathrm{Q}$ wave started and the $\mathrm{T}$ wave returned to the isoelectric line. The JT interval was derived by subtracting the QRS duration from the QT interval. QT and JT intervals were measured manually with calibers and a magnifying glass. Subjects with $\mathrm{U}$ waves on their ECGs were excluded from the study. All the measurements were repeated three times, and average values were accepted for each of the electrocardiographic parameters. QTd and JTd were defined as the difference between the longest and shortest QT and JT intervals; rate correction was performed with the Bazett's formula. ${ }^{13,15}$

Images were obtained on a Siemens Acuson Sequoia C256 cardiac ultrasonographic scanner (Acuson, Mountain View, CA, USA), with 2.5 to 3.5 $\mathrm{MHz}$ transducers. Patients were in the left lateral decubitus position, resting comfortably. M-mode tracings were obtained at the level of the mitral leaflets' tips in the parasternal long-axis position, and measurements of left ventricular end-systolic dimension and left ventricular end-diastolic dimension were taken according to the recommendations of the American Society of Echocardiography. ${ }^{9}$ In M-mode echocardiography, ventricular septal and posterior wall thicknesses were measured at end diastole through a parasternal long-axis view. Left ventricular ejection fraction and fractional shortening were obtained using the Teichholz method. ${ }^{9}$

\section{Statistical analysis}

Statistical analyses were performed by using SPSS version 15.0 statistical software (SPSS Inc., Chicago, IL, USA). Distribution of continuous variables was evaluated by one sample Kolmogorov-Smirnov test. As for descriptive statistics, mean \pm standard deviation was used for normally distributed variables, and median 
Table 1. Demographic and clinical features of FMF patients and healthy controls

\begin{tabular}{|c|c|c|c|c|c|c|c|}
\hline & \multicolumn{3}{|c|}{ FMF patients } & \multicolumn{3}{|c|}{ Healthy controls } & \multirow[b]{2}{*}{$p$} \\
\hline & $\mathrm{n}$ & $\%$ & Mean \pm SD & $\mathrm{n}$ & $\%$ & Mean \pm SD & \\
\hline Number of subjects & 48 & & & 31 & & & 0.906 \\
\hline Age (years) & & & $11.10 \pm 3.42$ & & & $9.61 \pm 2.83$ & 0.742 \\
\hline Gender & & & & & & & 0.684 \\
\hline Male & 26 & & & 17 & & & \\
\hline Female & 22 & & & 14 & & & \\
\hline Mean heart rate (beats/min) & & & $86.61 \pm 12.487$ & & & $84.42 \pm 12.863$ & 0.652 \\
\hline Systolic BP (mmHg) & & & $103.62 \pm 10.53$ & & & $102.85 \pm 11.67$ & 0.667 \\
\hline Diastolic BP (mmHg) & & & $66.78 \pm 10.22$ & & & $65.74 \pm 11.08$ & 0.096 \\
\hline C-reactive protein $(\mathrm{mg} / \mathrm{L})$ & & & $0.41 \pm 0.73$ & & & $0.33 \pm 0.15$ & 0.526 \\
\hline Sedimentation rate $(\mathrm{mm} / \mathrm{h})$ & & & $11 \pm 9.42$ & & & $8.96 \pm 6.42$ & 0.688 \\
\hline \multicolumn{8}{|l|}{ Clinical manifestations } \\
\hline Abdominal involvement & 37 & 77 & - & - & - & - & - \\
\hline Arthritic involvement & 7 & 15 & - & - & - & - & - \\
\hline Chest involvement & 10 & 21 & - & - & - & - & - \\
\hline Fever & 46 & 96 & - & - & - & - & - \\
\hline Duration of disease (months) & & & $48 \pm 5.2$ & - & - & - & - \\
\hline
\end{tabular}

(minimum-maximum) was used for not normally distributed continuous variables. Categorical variables were presented as frequencies and percentages. For continuous variables, an independent sample t-test and Mann-Whitney $\mathrm{U}$ test were used for independent samples comparisons. Chi-square test and Fischer's exact tests were used for comparisons of categorical variables. Correlation analyses were performed by using the Pearson correlation coefficient. A two-tailed $\mathrm{p}<0.05$ was considered statistically significant.

\section{RESULTS}

The duration of disease was $48 \pm 5.2$ months. None of the participants had a family history of FMF. According to the baseline clinical and demographic characteristics, the patients with
FMF and the control subjects were similar with regard to age and sex (Table 1). All patients and controls were in sinus rhythm and had normal 12-lead ECG at rest.

No statistically significant difference was detected between the FMF patients and healthy controls in terms of RR, QT, QTd, QTcd, JT, JTc, JTd, and JTcd measurements (Table 2). QTc value was higher in the FMF patients than in the healthy controls ( $\mathrm{t}=2916, \mathrm{p}=0.005)$; although the difference was statistically significant, the value is within normal limits (below 0.44).

Comparisons of the baseline echocardiographic values between patients and healthy controls are summarized in Table 3. There was no difference between the groups regarding left ventricular end-diastolic dimension, left ventricular end-systolic dimension, interventricular septum thickness

Table 2. Electrocardiographic measurements of FMF patients and healthy controls

\begin{tabular}{|c|c|c|c|c|}
\hline & FMF patients & Healthy controls & $\mathrm{t}$ & $p$ \\
\hline & Mean \pm SD & Mean \pm SD & & \\
\hline QT (ms) & $339.46 \pm 27.01$ & $329.81 \pm 32.64$ & 1.428 & 0.15 \\
\hline QTd (ms) & $28.54 \pm 9.89$ & $24.61 \pm 7.93$ & 1.858 & 0.06 \\
\hline QTc (ms) & $412.15 \pm 21.45$ & $393.58 \pm 35.18$ & 2.916 & 0.005 \\
\hline QTcd (ms) & $34.17 \pm 13.09$ & $30.00 \pm 13.67$ & 1.357 & 0.17 \\
\hline JT (ms) & $266.77 \pm 29.56$ & $258.39 \pm 31.70$ & 1.196 & 0.23 \\
\hline JTd (ms) & $29.27 \pm 10.91$ & $25.23 \pm 8.38$ & 1.755 & 0.08 \\
\hline JTc (ms) & $315.65 \pm 45.87$ & $316.55 \pm 25.62$ & 0.100 & 0.92 \\
\hline $\operatorname{JTcd}(\mathrm{ms})$ & $35.79 \pm 15.77$ & $32.48 \pm 13.32$ & 0.966 & 0.33 \\
\hline
\end{tabular}


Table 3. Comparison of echocardiographic parameters between FMF patients and healthy controls

\begin{tabular}{|c|c|c|c|c|}
\hline \multirow[b]{2}{*}{ Parameters } & \multirow{2}{*}{$\frac{\text { FMF patients }(\mathrm{n}=48)}{\text { Mean } \pm \text { SD }}$} & \multirow{2}{*}{$\frac{\text { Healthy controls }(\mathrm{n}=31)}{\text { Mean } \pm \text { SD }}$} & \multirow[t]{2}{*}{ Test value } & \multirow[t]{2}{*}{$p$} \\
\hline & & & & \\
\hline Left ventricle end-diastolic diameter (mm) & $38.20 \pm 4.47$ & $37.96 \pm 6.38$ & $\mathrm{t}=-0.187$ & 0.866 \\
\hline Left ventricle end-systolic diameter (mm) & $23.15 \pm 5.51$ & $22.86 \pm 4.76$ & $\mathrm{t}=-0.316$ & 0.742 \\
\hline Interventricular septum thickness in diastole $(\mathrm{mm})$ & $6.45 \pm 1.23$ & $6.14 \pm 1.66$ & $Z=-0.087$ & 0.820 \\
\hline Interventricular septum thickness in systole (mm) & $9.88 \pm 4.02$ & $9.75 \pm 3.49$ & $Z=-0.022$ & 0.988 \\
\hline Left ventricular posterior wall thickness in diastole $(\mathrm{mm})$ & $6.37 \pm 1.69$ & $6.34 \pm 1.77$ & $Z=-0.066$ & 0.969 \\
\hline Left ventricular posterior wall thickness in systole (mm) & $9.46 \pm 3.85$ & $9.67 \pm 3.22$ & $Z=-0.888$ & 0.380 \\
\hline Left atrium dimension $(\mathrm{mm})$ & $22.88 \pm 4.29$ & $23.05 \pm 4.45$ & $\mathrm{t}=-1.204$ & 0.399 \\
\hline Aortic dimension $(\mathrm{mm})$ & $19.67 \pm 3.32$ & $20.36 \pm 3.56$ & $Z=-1.016$ & 0.297 \\
\hline Ejection fraction & $70.49 \pm 4.89$ & $70.75 \pm 4.22$ & $t=0.385$ & 0.723 \\
\hline Fractional shortening & $39.52 \pm 4.18$ & $39.44 \pm 4.65$ & $\mathrm{t}=0.466$ & 0.683 \\
\hline
\end{tabular}

in diastole, interventricular septum thickness in systole, left ventricular posterior wall thickness in diastole, left ventricular posterior wall thickness in systole, left atrium dimension, aortic dimension, ejection fraction, and fractional shortening.

\section{DISCUSSION}

In autoimmune diseases, conduction disturbances and rhythm disorders are important manifestations of cardiac involvement, in addition to accelerated atherosclerosis. ${ }^{16,17}$ FMF is also associated with conduction disturbances and rhythm abnormalities, although with no important supporting evidence. ${ }^{18}$ The ventricular repolarization heterogeneity is an expression of regional differences in cellular action potential duration and ventricular recovery time. Variations in the duration of the action potential would create a ventricular repolarization dispersion that could be arrhythmogenic. ${ }^{13,19}$ QTd and JTd are an index in homogeneity of ventricular repolarization, and an important predictor of cardiovascular mortality in FMF patients similarly, as in other rheumatologic diseases. ${ }^{8}$

In recent years, arrhythmogenic tendency -particularly in the uncomplicated FMF disease- is controversial in some publications, and there is little evidence about it in relation to pediatric age group. Therefore, in this study, we included our assessment of QTd as well as JTd.

Akçay et al. ${ }^{8}$ reported that the FMF patients had a significantly higher QTd and higher QTcd relative to the controls and suggested that this dispersion was due to subclinical atherosclerosis and inflammatory features of FMF. ${ }^{6}$ In our study, inflammatory markers were normal in both groups.

Nussinovitch et al. ${ }^{20}$ reported that there was no statistically significant difference between the groups in terms of QT and QTd parameters (QTd: $48 \pm 12.5$ versus $46.7 \pm 9.7$ milliseconds; and QTcd: $51.4 \pm 12.0$ versus $49.7 \pm 10.5$ milliseconds). Furthermore, they reported that there is no significant difference in their similar studies of patients with FMF-amyloidosis, colchicine resistant-FMF, and FMF-responsive to colchicine, respectively. ${ }^{21-24}$ Similar to our study, Topal et al. ${ }^{25}$ did not find any significant difference between the 35 uncomplicated FMF patients and the 38 healthy controls in terms of QTd.

Similar to our results, Koca et al. ${ }^{6}$ demonstrated normal QTd in the pediatric age group. Moreover, Sahin et al. ${ }^{26}$ detected no statistical difference between children with FMF and healthy controls in regard to QT, QTc, QTd, and QTcd. In our study, while JT dispersion was not different between the two groups, QTc was higher in the FMF patients $(412.15 \pm 21.45)$ compared to the healthy controls (393.58 \pm 35.18$)$. Although it is in normal range (under $440 \mathrm{msn}$ ), QTc should be considered an important electrocardiographic data, indicating the sensitivity of ventricles. In another pediatric study, Arslan et al. ${ }^{27}$ found that $\mathrm{P}$-wave dispersion in children with FMF is higher than it is in the healthy controls. They suggested that $\mathrm{P}$-wave dispersion is related with increased arrhythmogenicity in children with FMF.

In another study, Kalkan et al. $^{28}$ performed conventional, strain, and tissue Doppler 
echocardiography in 23 FMF patients and 22 healthy controls and did not observe any significant differences between the FMF patients and healthy controls in terms of twodimensional and M-mode echocardiography, nor in conventional Doppler and tissue Doppler velocities. The authors only found that left ventricular strain values were significantly lower in FMF patients than in controls. ${ }^{26}$ In our study, we also detected similar conventional echocardiography values in FMF patients and controls.

The present study does have some limitations, primarily because of the small sample size as well as the lack of patient groups not receiving colchicine. Nevertheless, the results seem to be significant. As a consequence, patients with FMF who are continuously treated with regulardose colchicine, attack free, and have not developed amyloidosis appear to have similar QTd and JTd parameters and conventional echocardiography values as those of the healthy controls. Although FMF patients were not considered to be carrying significant potential cardiac risk with these results, QTc value may indicate increased ventricular sensitivity, and it is an important marker of cardiovascular mortality with considerable effect on sudden cardiac death and arrhythmia.

\section{Declaration of conflicting interests}

The authors declared no conflicts of interest with respect to the authorship and/or publication of this article.

\section{Funding}

The authors received no financial support for the research and/or authorship of this article.

\section{REFERENCES}

1. Saglam C, Polat A, Jones OY, Demirkaya E; FMF Arthritis Vasculitis and Orphan Disease Research in Paediatric Rheumatology (FAVOR). Recent advances in the management of children with familial Mediterranean fever. Int $\mathrm{J}$ Clin Rheumatol 2013;8:233-45.

2. Polat A, Demirkaya E, Basbozkurt G, Gattorno M, Ozen S; FMF Arthritis Vasculitis and Orphan disease Research in Paediatric Rheumatology (FAVOR). A glance at history and future perspectives of childhood autoinflammatory disorders. Ann Paediatr Rheum 2012;1:17-30.
3. Özçakar ZB, Yalçınkaya F. Why a new diagnostic criteria for pediatric Familial Mediterranean fever patients? Ann Paediatr Rheum 2012;1:207-13.

4. Caltik A, Akyuz SG, Erdogan O, Bulbul M, Orun UA, Demircin G. An unusual presentation of familial mediterranean fever with pericardial tamponade: report of two cases. Ann Paediatr Rheum 2012;1:190-4.

5. Caliskan M, Gullu H, Yilmaz S, Erdogan D, Unler GK, Ciftci O, et al. Impaired coronary microvascular function in familial Mediterranean fever. Atherosclerosis 2007;195:161-7.

6. Koca B, Kasapçopur O, Bakari S, Sönmez E, Oztunç F, Eroğlu AG, et al. QT dispersion and cardiac involvement in children with Familial Mediterranean fever. Cardiol Young 2012;22:404-9.

7. Bilginer Y, Ozaltin F, Basaran C, Duzova A, Besbas $\mathrm{N}$, Topaloglu R, et al. Evaluation of intima media thickness of the common and internal carotid arteries with inflammatory markers in familial Mediterranean fever as possible predictors for atherosclerosis. Rheumatol Int 2008;28:1211-6.

8. Akcay A, Acar G, Sayarlioglu M, Sokmen A, Kaya $\mathrm{H}$, Ispiroglu $\mathrm{M}$, et al. QT dispersion and transmural dispersion of repolarization in patients with familial Mediterranean fever. Mod Rheumatol 2009; 19:550-5.

9. Lang RM, Bierig M, Devereux RB, Flachskampf FA, Foster E, Pellikka PA, et al. Recommendations for chamber quantification: a report from the American Society of Echocardiography's Guidelines and Standards Committee and the Chamber Quantification Writing Group, developed in conjunction with the European Association of Echocardiography, a branch of the European Society of Cardiology. J Am Soc Echocardiogr 2005;18:1440-63.

10. Barr CS, Naas A, Freeman M, Lang CC, Struthers AD. QT dispersion and sudden unexpected death in chronic heart failure. Lancet 1994;343:327-9.

11. Amlie JP. QT dispersion and sudden cardiac death. Eur Heart J 1997;18:189-90.

12. Zabel M, Portnoy S, Franz MR. Electrocardiographic indexes of dispersion of ventricular repolarization: an isolated heart validation study. J Am Coll Cardiol 1995;25:746-52.

13. Hacihamdioglu DO, Fidanci K, Kilic A, GokF, Topaloglu R. QT and JT dispersion and cardiac performance in children with neonatal Bartter syndrome: a pilot study. Pediatr Nephrol 2013;28:1969-74.

14. Livneh A, Langevitz P, Zemer D, Zaks N, Kees $\mathrm{S}$, Lidar $\mathrm{T}$, et al. Criteria for the diagnosis of familial Mediterranean fever. Arthritis Rheum 1997;40:1879-85.

15. Bazett HC. An analysis of the time relations of electrocardiograms. Heart 1920;7:353-67.

16. Knockaert DC. Cardiac involvement in systemic inflammatory diseases. Eur Heart J 2007;28:1797804. 
17. Abu-Shakra M, Urowitz MB, Gladman DD, Gough J. Mortality studies in systemic lupus erythematosus. Results from a single center. I. Causes of death. J Rheumatol 1995;22:1259-64.

18. Gale A, Levin ME. Pericarditis and auricular fibrillation. Occurrence in familial mediterranean fever. Arch Intern Med 1963;112:234-41.

19. Tomaselli GF, Beuckelmann DJ, Calkins HG, Berger RD, Kessler PD, Lawrence JH, et al. Sudden cardiac death in heart failure. The role of abnormal repolarization. Circulation 1994;90:2534-9.

20. Nussinovitch N, Livneh A, Katz K, Langevitz $P$, Feld O, Nussinovitch $\mathrm{M}$, et al. QT dispersion in uncomplicated familial Mediterranean fever. Clin Rheumatol 2010;29:1353-6.

21. Nussinovitch $U$, Nussinovitch $N$, Nussinovitch $M$, Volovitz B, Feld O, Ben-Zvi I, et al. QT dispersion in amyloidosis due to familial Mediterranean fever. Rheumatol Int 2012;32:1945-8.

22. Nussinovitch U, Kaminer K, Nussinovitch M, Volovitz B, Lidar M, Nussinovitch $\mathrm{N}$, et al. QT interval variability in familial Mediterranean fever: a study in colchicine-responsive and colchicine-resistant patients. Clin Rheumatol 2012;31:795-9.
23. Nussinovitch U, Livneh A, Volovitz B, Nussinovitch M, Ben-Zvi I, Lidar M, et al. Normal QT dispersion in colchicine-resistant familial Mediterranean fever (FMF). Clin Rheumatol 2012;31:1093-6.

24. Nussinovitch U, Ben-Zvi I, Livneh A. QT variability in amyloidosis of familial Mediterranean fever. Isr Med Assoc J 2012;14:225-8.

25. Topal F, Tanindi A, Kurtoglu HG, Akbulut S, Kucukazman M, Topal FE. QT dispersion is not increased in familial Mediterranean fever. $\mathrm{J}$ Int Med Res 2011;39:2006-11.

26. Sahin M, Kır M, Makay B, Keskinoğlu P, Bora E, Unsal $E$, et al. Cardiac autonomic functions in children with familial Mediterranean fever. Clin Rheumatol. 2014 Jun 15. [Epub ahead of print]

27. Arslan D, Oran B, Yazılitas F, Peru H, Cimen D, Vatansev H. P-wave duration and dispersion in children with uncomplicated familial Mediterranean fever. Mod Rheumatol 2013;23:1166-71.

28. Kalkan GY, Bayram NA, Erten S, Keles T, Durmaz T, Akcay M, et al. Evaluation of left ventricle function by strain imaging in patients with familial Mediterranean fever. Echocardiography 2010;27:1056-60. 